$\xi=-1$

\title{
Iaas: Qos based Automated User Requirement Identification for Optimal Resource Allocation in Multi Cloud
}

\author{
${ }^{1}$ K.S.Guruprakash, ${ }^{2}$ Syed Fiaz.A.S, ${ }^{3}$ S.Sankar \\ ${ }^{1,2,3}$ Assistant Professor, Department of Computer Science and Engineering \\ ${ }^{1,2}$ Veltech University, Chennai, Tamilnadu, India \\ ${ }^{3}$ Dhirajlal Gandhi College of Technology, Salem, Tamilnadu, India
}

\begin{abstract}
Resource allocation and optimization is one of the important characteristics in cloud computing environment. An vital goal of any cloud service provider is to allocate cost effective and optimized resource packages to the consumers that meet the QOS (Quality of Service) requirements. Though the various set of cloud resources are available, selecting an appropriate resource to the consumers based on their requirements is a tedious task for any providers. Many researchers have already discussed different algorithms for finding the optimal resources to the consumers. However there is a challenge in selecting exact resources that meet QoS Parameters such as performance, availability, reliability and so on. This paper proposes an effective method for optimal resource allocation in multi cloud environment. This approach takes an input, set of QOS parameter value for each user and select the suitable package that matches the QOS value. This paper provides an effective resource allocation solution to IaaS (Infrastructure as a Service) provider based on consumers usage patterns.
\end{abstract}

Keywords: Cloud Resource allocation, Cloud Service Recommendation, Optimal Resource package allocation, Cloud Compitting, Cloud Infrastructure

\section{Introduction}

Cloud Computing is a model which provides computing resources on demand as pay as you go. Various services provided by cloud service provider are Software As a Service (SaaS), Infrastructure as a Service (IaaS) and Platform as a Service (PaaS). Cloud Computing is mainly focused on delivering an efficient and flexible virtual services to the users from everywhere by satisfying users demand at low cost. Because of this benefit, many organizations migrate from web to cloud for providing services to their business clients. Cloud Infrastructure consists of diverse resources being utilized for deploying large scale distributed applications based on different users needs. In IaaS, application provider get the requirements from the consumers and choose the appropriate service provider that meet the consumers need to achieve the QoS. Here application providers are the entities who host the application on the cloud resource provided by the cloud infrastructure service provider. Consumers are the entities who use the application hosted by application provider. The general model of cloud infrastructure service is shown in Figure 1. The main objective here is to allocate sufficient number of resources to the consumers based on their need. At the same time, service provider ensures that no excess number of resources provided to the consumers. If excess number of resources provided to consumers, it will reduce the revenue of the cloud resource providers as well as consumers have to pay for the unused resources. This is what the over utilization or underutilization of resources. It is a major problem in cloud computing. Considering the IaaS cloud services, the fundamental requirements are that users should state their requirements for allocation of appropriate resources from IaaS Providers. However at an early stage, most of the users are not aware of exact requirements to be specified. This increases the requirements frequently from time to time which further increase the cost of the consumers. Usually, cloud resources are not available as complete for user to access the services. Instead, resources are provided as a package with different configuration. Appropriate resource packages are allocated to users according to their need. Packages are provided to consumers with different level of QOS parameter values such as performance, reliability, storage, availability, robustness and so on. Package selection plays an important role in satisfying users need. The major problem faced during package selection process is to select the suitable package that matches the user's current requirements. To solve this problem, system should be automated instead of getting requirements manually. Automation process is not taken by any cloud providers in the traditional system. Only few authors have been discussed on automated user requirements identification for cloud service recommendation [13] This leads to select inappropriate packages for the consumers which further leads underutilization or over utilization of resources. This is another drawback of an existing system without automation. Automation process use cloud based $\log$ file for extracting the requirements of consumers based on their usage pattern. Once the requirements are extracted from log file, input request file is prepared. An input request file along with additional workload and package files are fed into optimizer for choosing the best suitable resources among the resource packages from suitable service provider.

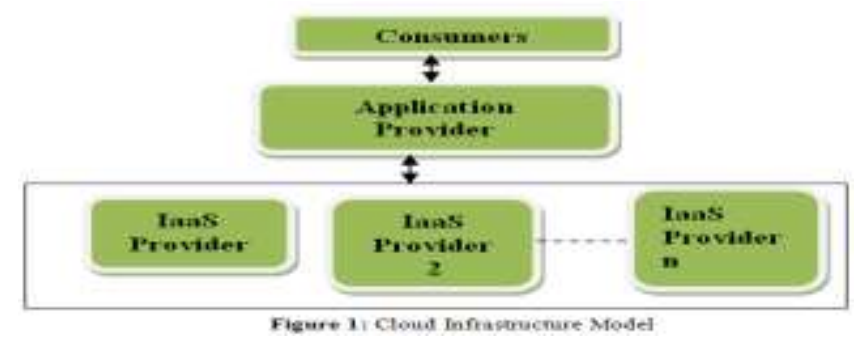


The rest of the paper discussed the following sections. Section 2 reveals the related work of resource allocation and optimization techniques. Section 3 discussed the proposed model of how to perform automated user requirement identification and QOS Parameter ranking. Section 4 discussed the package selection algorithm for IaaS users. Section 5 discussed the conclusion and future work.

\section{Related Work}

Yu sun et.al [1] proposed cost effective model based system that simplifies, optimize and automate the cloud resource allocation that meets the users need. This system performs automatic load and resource testing for the web applications and allocates the cost effective resources to the consumers. Ferry et.al [2] discussed the overview of different model based techniques for cloud resource optimization. This technique proposed architecture for multi cloud system. Another QOS based modeling framework for automated cloud resource allocation and optimization was proposed by $\mathrm{Yu}$ sun et.al[3] which automatically perform load testing for complex multi tier distributed application in cloud. This technique also provides optimization technique for allocating cloud resources. Draheim et.al [5] proposed a new method for allocate resource based on real users behavior. Load Testing Framework (LTF) was proposed by Wang et.al [4] which models users behavior that performs load testing in different workflow and provide resources as per the need.

Automatic resource allocation technique was proposed by S.Marrone et.al [6] which lead cloud brokers to find optimal resource configuration. This approach use Bayesian network to optimize cloud resources..Gupta et.al[7] proposed resource allocation technique that concentrates on acquiring efficient energy. In this technique, energy utility parameters are considered for allocating efficient resources to the consumers. Bahrpeyma et.al [8] proposed technique to improve the elasticity in the cloud environment which used to allocate resources dynamically.

K.S.Guruprakash et.al [13] proposed method for identifying user requirements from users log file for resource recommendation in cloud. Resource allocation based on user requirements was proposed by Madhumathi [14] which used ACO (Ant colony Optimization) algorithm for selecting resource package to consumers. This method provides effective service for only academic data sets.

In all the above methods, there are only few author considered automatic user requirements identification.

All the authors mostly concentrated on single cloud environment However none of the author focused on multi cloud environment. This paper proposed the work for application provider to choose the best IaaS service provider among the multiple providers Selecting the best resource package among the multi cloud service provider and selecting the best service provider among the multiple service providers are another challenging tasks in cloud environment. The remaining sections discussed how to achieve package selection effectively in multi cloud environment for IaaS users.

\section{Proposed Model}

The proposed model is shown in Figure 2. This model takes users $\log$ file as an input and extracts some of the QoS attributes from $\log$ file. This QoS attributes indicates the user's requirements. IaaS users also rank the QoS parameter by giving their own weightage. QoS value identified from log file and Weighted QoS value are combined to form final QoS value. Based on the final QoS value, user requirement file is created and fed into optimizer running in QoS mapper. The optimizer choose an appropriate package from suitable IaaS service provider according to the users requirements.

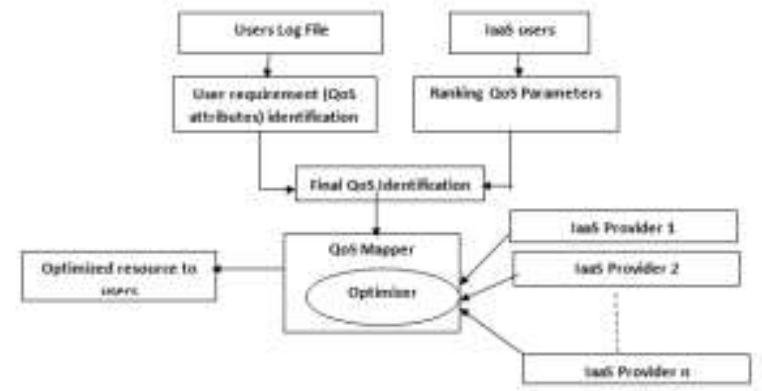

Figure 2: Proposed Model for optimized package selection in multi cloud

\subsection{Automated User Requirement Identification}

Cloud service provider needs some QoS parameters to provide resource allocation effectively. In the current system, requirements are specified manually by the users. Most of the users are not aware of requirements to be specified at initial configuration stage. Hence the requirements can be fluctuated which increase the cost of consumers because of either over utilization or under utilization of resources. To overcome this issue, user requirements are to be extracted automatically. This automation process is accomplished by users $\log$ file. Log file contains the usage patterns of utilizing resources. When user migrate from web to cloud for the first time, there is no log data for the corresponding user. At that time, user requirements are to be specified manually. Once the log file available for user, it can be used as a base for identifying exact requirements for the users which can avoid the problem of over utilization or under utilization of resources. The format for log file is shown in the Figure 3 .

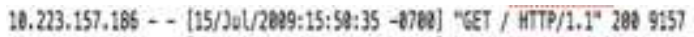

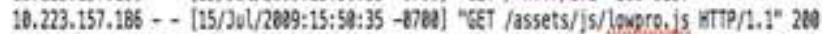 12469 \\ 10.223.157.185 - - [15/3uL/2629:15:59:35 - 8720$]$ "6Et /assets/css/reset.css HTTP/1.1" 206 1014

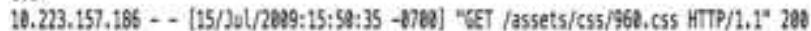 6226

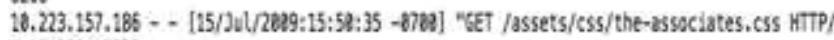 1.1220015779

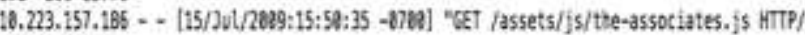 $1.1^{*} 2204492$}

Figure 3: Sample User access Log file

The above log file contains zn IP Address of the host which initiate request to server, time the server finished processing, protocol with request method, status code and size of the object returned to user.

This log file is taken as an input for our process and extracts the QoS Parameters that meet the users need. Only few QoS Parameters such as latency, usability, bandwidth, computational capacity, availability, correctness can be extracted from log file. Other parameters such as security, portability, reliability, backup data and customization have to be specified manually. The following formula can be used for extracting QoS Parameters from $\log$ file.

Bandwidth is calculated using

$B=b_{n} / T_{u}$ Where $b_{n}$ is no of bits sent or received, $T_{u}$ is Unit of Tine in seconds.

Computational capacity is calculated using

$\mathrm{CC}=\mathrm{U}_{\mathrm{a}} / \mathrm{U}_{\mathrm{e}}$ Where $\mathrm{U}_{\mathrm{a}}$ is Actual Usage time of resources, $\mathrm{U}_{\mathrm{e}}$ is Expected usage time of resources.

Usability is calculated using

$\mathrm{U}=\mathrm{N}_{\mathrm{s}} / \mathrm{T}$ Where $\mathrm{N}_{\mathrm{s}}$ is No of successful operations. T is Total no of operations

Correctness can be calculated using

$\mathrm{C}=\mathrm{T}_{\mathrm{f}} / \mathrm{T}_{\mathrm{s}}+\mathrm{T}_{\mathrm{f}}$ Where $\mathrm{T}_{\mathrm{f}}$ is Total no of failed tranQoSssion, $\mathrm{T}_{\mathrm{s}}$ is Total no of successful tranQoSssion.

Latency can be calculated using

$\mathrm{L}=\mathrm{T}_{\mathrm{i}}-\mathrm{T}_{\mathrm{o}}$ Where $\mathrm{T}_{\mathrm{i}}$ is Input time, $\mathrm{T}_{\mathrm{o}}$ is Output time. 
Availability can be calculated using

$\mathrm{A}=$ MTTF / MTTF + MTTR Where MTTF is mean time to failure and MTTR is mean time to repair.

This phase identifies some of the QoS Parameters based on the users $\log$ file.

\subsection{Ranking Qos Parameters}

The identified QoS Parameter in previous phase helps to find the users requirement. The next phase is to rank QOS Parameter based on the user's weightage. This ranking is required to identify the requirement levels of resource packages and its fitness. In order to rank QOS Parameter, Users have to assign weight based on their preference. This assignment can be done by comparing one attributes with other attributes. For some attributes, users can directly assign weights. QoS value should be normalized since the value identified is in various ranges. Using the normalized QoS value and weights obtained, actual user requirements can be estimated using weighted sum method.

$$
\sum_{i=1}^{n} Q_{i}^{*} \boldsymbol{W}_{\boldsymbol{i}} \text { Where } \mathrm{n} \text { is no of QoS Parameter }
$$

Qi is normalized QoS value, Wi is weight assigned by user based on their preference. Now the final QoS requirement is obtained.

\section{Optimized Resource Package Selection in Multi Cloud}

After determine the final QoS requirements, user requirements package file is created with final QoS value. User requirement file is then passed to QoS Mapper which performs optimization through optimizer. Each IaaS provider has their own package file. Optimization is run on each package file to choose the appropriate packages from different service provider. Now all the selected packages are combined and run the optimizer again to choose the suitable package from best service provider that matches the users need.

\section{Conclusions and Future Work}

Cloud resource allocation and optimization is one of the important challenge in cloud computing environment. In order to allocate appropriate resource effectively to the consumers, identification of an exact requirements play a vital role for Cloud service provider (For IaaS) that meet QoS. To accomplish this, users log file is taken as an input and QoS requirements are identified. Based on the requirements identified, QoS Mapper selects the corresponding service provider and the appropriate packages that matches the requirements. This paper provides effective solutions to the IaaS service provider to achieve Quality of Service (QoS). Our future work is to improve the Quality of Service by considering the elasticity scaling in the cloud environment. We can extend the future work by taking the cost factors for effective package selection in multi cloud environment.

\section{References}

[1] Y. Sun, J. White, S.Eade, and D.C. Schmidt, "ROAR: A QoSoriented modeling framework for automated cloud resource allocation

[2] and optimization",Journal of Systems and Software, 116, 146-161, 2016.

[3] N.Ferry, A. Rossini, F.Chauvel, B. Morin, "A Solberg Towards model-driven pro- visioning, deployment, monitoring, and adaptation

[4] of multi-cloud systems,In: Pro- ceedings of the IEEE 6th International Conference on Cloud Computing. 887-894, 2013.

[5] Yu Sun, Jules White, Sean Eade "A Model-Based System to Automate Cloud Resource Allocation and Optimization",
International Conference on Model Driven Engineering Languages and Systems, pp 18-34, 2014.

[6] X. Wang, B. Zhou, andW. Li, "Model-based load testing of web applications",J. Chin. Inst. Eng. 36 (1), 74-86, 2013.

[7] D. Draheim, J. Grundy, J. Hosking, C.Lutteroth, andG. Weber, "Realistic load test- ing of web applications", In: Proceedings of the

[8] IEEE 10th European Conference on Software Maintenance and Reengineering, CSMR. IEEE, 11, 2006

[9] S. Marrone, andR. Nardone, "Automatic resource allocation for high availability cloud services",Procedia Computer Science, 52,

[10] 980-987, 2015

[11] P. Gupta, andS.P. Ghrera, "Power and Fault Aware Reliable Resource Allocation for Cloud Infrastructure",Procedia Computer Science. 78, 457-463, 2016

[12] F. Bahrpeyma, H.Haghighi, andA. Zakerolhosseini, "An adaptive RL based approach for dynamic resource provisioning in Cloud virtualized data centers", Computing, 97(12), 1209-1234, 2015.

I. Foster, Y. Zhao, I. Raicu, S. Lu,"Cloud computing and grid computing 360-degree compared",In: Grid computing environments workshop (GCE'08). 1-10, 2008.

[13] M. Armbrust, A. Fox, R. Griffith, A.D. Joseph, R.H. Katz, A.Konwinski, G. Lee, D.A. Patterson, A.Rabkin, I.Stoica, and M. Zaharia,"Above the clouds: A berkeley view of cloud computing", 2009.

[14] H. Hayes, "Cloud computing", Commun ACM. 51, 9-11, 2008.

[15] C. Li, "Optimal resource provisioning for cloud computing environment", The Journal of Supercomputing. 62(2), 989-1022, 2016

[16] K.S.Guruprakash, Sy.Siva Sathya "Log based Automated SMI Parameter Identification and Resource recommendations in cloud" Indian Journal of Science and Technology, Vol 9(30), DOI: 10.17485/ijst/2016/v9i30/99011, August 2016

[17] C. Madhumathi, andG. Ganapathy, "Requirement Intensity Based Resource Provisioning For E-Learning In Multi-Cloud To Avoid Vendor Lock-Ins",ARPN Journal of Engineering and Applied Sciences, Vol.11, No17, 2016. 